\title{
The effect of silver nanoparticles on the reproductive system of adult male rats: A morphological, histological and DNA integrity study
}

\author{
Nasibeh Fathi 1,B,D, Seyed Mohammad Hoseinipanah ${ }^{1, A}$, Zohreh Alizadeh 2,B, Mohammad Javad Assari, B,B, , \\ Abbas Moghimbeigi ${ }^{4, C}$, Motahare Mortazavi ${ }^{5, B}$, Morteza Haji Hosseini ${ }^{6, C}$, , Maryam Bahmanzadeh ${ }^{1,2, A-F}$ \\ ${ }^{1}$ Department of Anatomical Sciences, School of Medicine, Hamadan University of Medical Sciences, Iran \\ 2 Endometrium and Endometriosis Research Center, Hamadan University of Medical Sciences, Iran \\ ${ }^{3}$ Center of Excellence for Occupational Health, Occupational Health and Safety Research Center, School of Public Health, Hamadan University of Medical Sciences, Iran \\ ${ }^{4}$ Department of Biostatistics and Epidemiology, School of Public Health, Hamadan University of Medical Sciences, Iran \\ ${ }^{5}$ Medicinal and Natural Products Chemistry Research Center, Shiraz University of Medical Sciences, Iran \\ ${ }^{6}$ Birjand Cardiovascular Diseases Research Center, Birjand University of Medical Sciences, Iran \\ A - research concept and design; B - collection and/or assembly of data; C - data analysis and interpretation; \\ $D$ - writing the article; $E$ - critical revision of the article; $F$ - final approval of the article
}

\section{Address for correspondence \\ Maryam Bahmanzadeh \\ E-mail: bahmanzadeh12@yahoo.com}

Funding sources

The study was funded by vice-chancellor for

Research and Technology, Hamadan University

of Medical Sciences, Iran (No. 9205011568).

Conflict of interest

None declared

\section{Acknowledgements}

This study has been adapted from an MSc thesis at Hamadan University of Medical Sciences, Iran. The authors would like to thank Dr. Hajilui for his technical assistance.

Received on September 1, 2016

Reviewed on March 25, 2017

Accepted on December 28, 2017

Published online on August 29, 2018

Cite as

Fathi N, Hoseinipanah SM, Alizadeh Z, et al. The effect of silver nanoparticles on the reproductive system of adult male rats: A morphological, histological and DNA integrity study. Adv Clin Exp Med. 2019;28(3):299-305. doi:10.17219/acem/81607

DOI

10.17219/acem/81607

\section{Copyright}

Copyright by Author(s)

This is an article distributed under the terms of the Creative Commons Attribution Non-Commercial License (http://creativecommons.org/licenses/by-nc-nd/4.0/)

\section{Abstract}

Background. Silver nanoparticles (AgNPs) are more often used in various products, and consequently the potential deleterious effects associated with exposure to them are of concern. Several lines of evidence have demonstrated that the toxicity of AgNPs affects different organs and leads to some side effects, including weight loss, inflammation and cell death.

Objectives. The aim of this study was to evaluate the effect of different concentrations of AgNPs on sperm parameters and testicular histology.

Material and methods. In the present study, 28 male adult Wistar rats were categorized into a control group and 3 experimental groups (AgNP-1, AgNP-2 and AgNP-3), intraperitoneally (i.p.) receiving 30, 125 and $300 \mathrm{mg} / \mathrm{kg}$ of AgNPs, respectively. Twenty-eight days after injection the epididymes and the testes of each rat were dissected in order to evaluate sperm parameters, sperm chromatin integrity and histomorphometric changes in the testicular tissue.

Results. The results showed a significant decrease in sperm count $(p<0.0001)$, vitality $(p<0.05)$ and morphology changes $(p<0.001)$ in the group receiving $300 \mathrm{mg} / \mathrm{kg}$ of AgNPs compared to the control group. A significant decrease was also observed in the number of spermatogonia, Sertoli and Leydig cells in the AgNP-2 and AgNP-3 groups ( $p<0.05)$. The evaluation of sperm chromatin did not show any significant differences among the experimental groups ( $p>0.05)$.

Conclusions. The data showed some dose-dependent adverse effects of AgNPs on sperm and seminiferous tubules. More experimental investigations are necessary to draw better conclusions regarding the safety of nanoparticles (NPs) on the male reproduction system.

Key words: testis, sperm chromatin, sperm parameters, histomorphometry, silver nanoparticles 


\section{Introduction}

Silver nanoparticles (AgNPs) are clusters of silver atoms (varying from 1 to $100 \mathrm{~nm}$ in diameter) that are used as antibacterial and antimicrobial agents in clinical medicine. Silver nanoparticles are integrated into various food-contact materials, such as plastics used to manufacture food containers, refrigerator surfaces, storage bags, and chopping boards, and are used to preserve foods by inhibiting microorganism growth. ${ }^{1,2}$ Evidence has shown that AgNPs have a potent anti-inflammatory effect. ${ }^{3}$ Silver nanoparticles are of great importance in the treatment of diseases. ${ }^{4}$

Regardless of their widespread utility, the influence of AgNPs on human health and the mechanisms of their action are not fully understood. Therefore, it is important to survey their potential toxicity in living organisms, especially in mammals, in order to provide a reliable human risk assessment. ${ }^{4}$ Nanoparticles (NPs) like silver can induce reactive oxygen species (ROS) production, which causes oxidative stress and toxicity in various cell types. ${ }^{5,6}$ Silver nanoparticles can pass through cell membranes, the bloodbrain barrier and the blood-testis barrier. ${ }^{7}$ The main target organs for AgNP deposition after systemic availability are the spleen, liver and kidneys, while there is less distribution to other organs. Additionally, high levels of silver have sometimes been found in the testes. ${ }^{5,6}$

The effects of AgNPs on testis and sperm function have been reported. Pothuraju and Kaul reported the effects of these NPs on buffalo sperm parameters, which showed a dose-dependent decrease in sperm viability without a change in sperm motility at a concentration of $50 \mathrm{mg} / \mathrm{kg} .{ }^{8}$

However, NPs must be investigated on a case-by-case basis to determine whether an NP will have a positive or negative effect on spermatogenesis, because it is dependent on the chemical composition, size, treatment period, dose level, route, duration of exposure, and recovery time., ${ }^{9,10}$

Therefore, the main purpose of the present study was to evaluate the effect of different concentrations of AgNPs on sperm parameters and chromatin, and testicular histomorphometry in rats.

\section{Material and methods}

\section{Nanoparticle preparation for analysis}

We prepared AgNPs as described previously. ${ }^{11}$ Silver nanoparticles were purchased from US Research Nanomaterials, Inc. (Houston, USA). Based on the specifications provided by the manufacturer, the AgNPs were of $99 \%$ purity as a nanopowder, with a nominal diameter of 60-80 nm. The AgNPs were suspended by sonication in deionized water (TKA Fisher Scientific Smart2Pure 30123; Thermo Fisher Scientific, Burladingen, Germany), and then diluted to concentrations of 30,125 and $300 \mathrm{mg} / \mathrm{kg}$ at the time of injection.

\section{Nanoparticle characterization}

The size distribution of AgNPs was measured by dynamic light scattering (DLS) (Zetasizer Nano ZS apparatus ZEN 3600; Malvern Panalytical, Malvern, UK) at $25^{\circ} \mathrm{C}$ and was started 2 min after the cuvette was placed in the DLS apparatus to reach thermal equilibrium. Measurements were conducted $24 \mathrm{~h}$ after the suspensions were prepared. ${ }^{11,12}$ A Philips-EM 208 transmission electron microscopy (TEM) and a scanning electron microscope (SEM) (Philips, Amsterdam, the Netherlands) were also employed to monitor the size and morphological changes in AgNPs.

\section{Animals}

Twenty-eight adult male Wistar rats weighing 200-250 g were kept under standard conditions (12-hour light/dark cycle at $22-24^{\circ} \mathrm{C}$, with free access to water and food). All of the procedures were carried out with minimal stress and discomfort in accordance with national guidelines and protocols approved by the Institutional Animal Ethics Committee of Hamadan University of Medical Sciences, Iran.

\section{Experimental design}

The animals were weighed and randomly divided into 4 groups ( $n=7$ per group): a control group and 3 experimental groups (AgNP-1, AgNP-2 and AgNP-3), receiving a single intraperitoneal (i.p.) dose of AgNPs at 30, 125 and $300 \mathrm{mg} / \mathrm{kg}$ concentrations, respectively. The animals in the control group were injected with distilled water. The animals were sacrificed 4 weeks after the injection. In rats, the duration of the spermatogenesis cycle is approx. 48-56 days. A half of this cycle and 2 cycles of the seminiferous epithelium takes 28 days. $^{10,13}$

\section{Sperm collection and count}

After 28 days, the left cauda epididymis of each animal was dissected and placed in a Petri dish containing Ham's F10 medium (Sigma-Aldrich, St. Louis, USA). The dishes were placed in the incubator for $15 \mathrm{~min}$. Approximately $10 \mu \mathrm{L}$ of the diluted sperm suspension was transferred to each counting chamber of a hemocytometer and allowed to stand for $5 \mathrm{~min}$. The cells which settled during this time were counted by a light microscope (Zeiss, Munich, Germany) at $\times 200$ magnification..$^{13}$

\section{Sperm morphology}

The sperm morphology was also determined as described previously. ${ }^{14}$ Morphological abnormalities were classified as headless sperm, a flattened head, a reduced hook or banana head, a pin or nail head, a bent neck, a bent tail, a kinked tail, and multiple abnormalities. The percentage 
of normal morphology of 100 spermatozoa per rat was assessed by light microscopy.

\section{Sperm motility}

Aliquots of the sperm suspension prepared for analysis were placed on a slide. The spermatozoa were classified as motile or immotile. The percentage of motility was evaluated for each of the animals used. ${ }^{15}$

\section{Sperm viability}

The sperm viability was also determined using eosin stain as described before. ${ }^{14}$ Eosin penetrated non-viable, dead spermatozoa with disrupted membranes, which appeared stained in red.

\section{Histological evaluation}

The left testes were removed and fixed in a 10\% buffered formalin solution for 2 weeks, and were then embedded in paraffin. The paraffin blocks were cut into 5-micrometer slices. The sections (3 per animal) were stained with hematoxylin and eosin (H\&E).$^{10}$ Twenty-five seminiferous tubules from each testis were randomly evaluated on circular cross-sections, and the spermatogonia, spermatocyte, spermatid, and Leydig and Sertoli cells were counted.

\section{Parameters of seminiferous tubules}

For each set of 25 seminiferous tubules from each testis, the parameters, including area, circumference and diameter, were determined under a light microscope equipped with Motic Moticam 2000 2.0M Pixel camera (Motic, Kowloon, Hong Kong). ${ }^{10}$

\section{Evaluation of sperm nuclear chromatin}

For the evaluation of sperm nuclear chromatin, 3 different techniques were applied.

\section{Aniline blue staining}

Aniline blue (AB) selectively stains lysine-rich histones and has been used for distinguishing sperm chromatin condensation anomalies. For this purpose, air-dried smears were prepared from fresh sperm samples of each rat and fixed in $3 \%$ buffered glutaraldehyde in a $0.2 \mathrm{M}$ phosphate buffer ( $\mathrm{pH} 7.2$ ) for $30 \mathrm{~min}$ at room temperature. Each smear was stained with $5 \%$ aqueous $\mathrm{AB}$ stain in $4 \%$ acetic acid ( $\mathrm{pH} 3.5$ ) for $5 \mathrm{~min}$. For light microscopy evaluation, 100 spermatozoa were counted in each slide and pale-blue spermatozoa were considered normal, while dark-bluestained ones were treated as abnormal spermatozoa. ${ }^{13}$

\section{Toluidine blue staining}

Toluidine blue (TB) is a metachromatic dye which measures the rate of sperm nuclear chromatin condensation via binding to phosphate groups of DNA strands. For this staining, air-dried sperm smears were fixed in fresh $96 \%$ ethanol:acetone $(1: 1)$ at $4^{\circ} \mathrm{C}$ for $30 \mathrm{~min}$, and then hydrolyzed in $0.1 \mathrm{~N}$ hydrochloric acid $(\mathrm{HCl})$ at $4^{\circ} \mathrm{C}$ for $5 \mathrm{~min}$. Next, the slides were rinsed thrice in distilled water for $2 \mathrm{~min}$, and finally stained with $0.05 \% \mathrm{~TB}$ for $10 \mathrm{~min}$. The component of staining buffer was $50 \%$ citrate phosphate ( $\mathrm{pH} 3.5){ }^{16}$ For light microscopic assessment, using $\times 100$ magnification, the chromatin quality of the spermatozoa was determined according to metachromatic staining of sperm heads in light blue (TB-) and purple (TB+).

\section{Acridine orange staining}

Acridine orange $(\mathrm{AO})$ is a fluorescence probe for measuring the susceptibility of sperm nuclear DNA to in situ acid-induced denaturation. Sperm DNA integrity was determined by AO staining. For this purpose, the smears were first air-dried and then fixed overnight in Carnoy's solution (methanol:glacial acetic acid, 3:1). Each sample was stained for $10 \mathrm{~min}$ in freshly prepared AO $(0.19 \mathrm{mg} / \mathrm{mL})$ in citrate phosphate buffer ( $\mathrm{pH}$ 2.5) for $10 \mathrm{~min}$. The smears were evaluated on the same day with an Olympus fluorescence microscope (Zeiss) with a 460-nanometer filter. The duration of illumination was limited to $40 \mathrm{~s}$ per field. The percentage of green (normal double-stranded DNA) and orange/red (abnormally denatured DNA) fluorescence spermatozoa per sample was calculated. ${ }^{16}$

\section{Statistical analysis}

Statistical analysis was performed using SPSS v. 16 (SPSS Inc., Chicago, USA) and the variables were analyzed by oneway analysis of variance (ANOVA), the Kruskal-Wallis test and the Mann-Whitney U test. The Tukey method was used for the following multiple comparison tests. All data was expressed as mean \pm standard deviation (SD). The statistical level of significance was set at $\mathrm{p}<0.05$.

\section{Results}

\section{Characterization of silver nanoparticles}

As previously mentioned, the DLS analysis demonstrated a broad hydrodynamic diameter peak with an average size of $250 \mathrm{~nm}$ (nominally $<100 \mathrm{~nm}$ in diameter, but actually $\sim 250 \mathrm{~nm}$ ) (Fig. 1). ${ }^{11}$ The diameters of NPs observed under TEM were in agreement with the DLS results (Fig. 2). ${ }^{17}$ According to the SEM micrographs, the AgNPs were almost spherical (Fig. 3), but had a tendency to agglomerate or aggregate in the solution. 


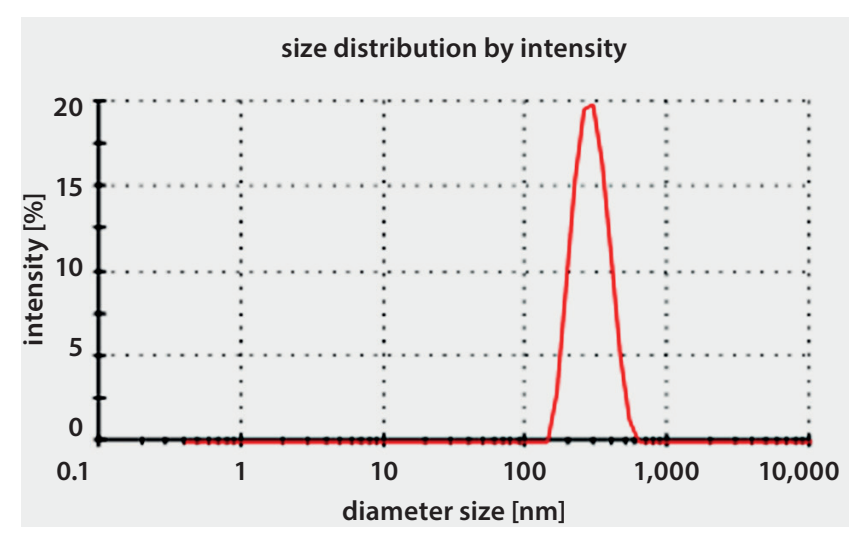

Fig. 1. Dynamic light scattering results of the characterization of nanosilver used in the study

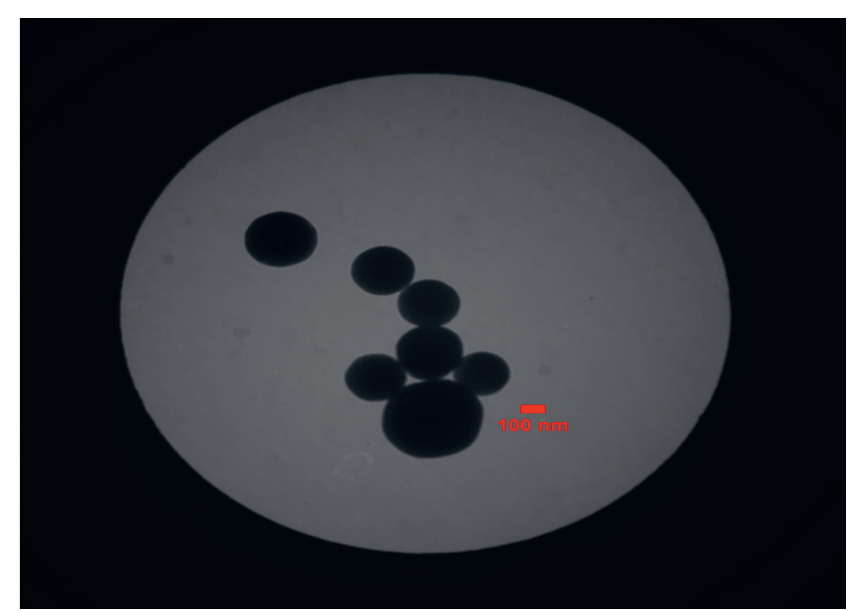

Fig. 2. Transmission electron microscopy (TEM) image of silver nanoparticles (AgNPs); scale bars indicate $10 \mathrm{~nm}$

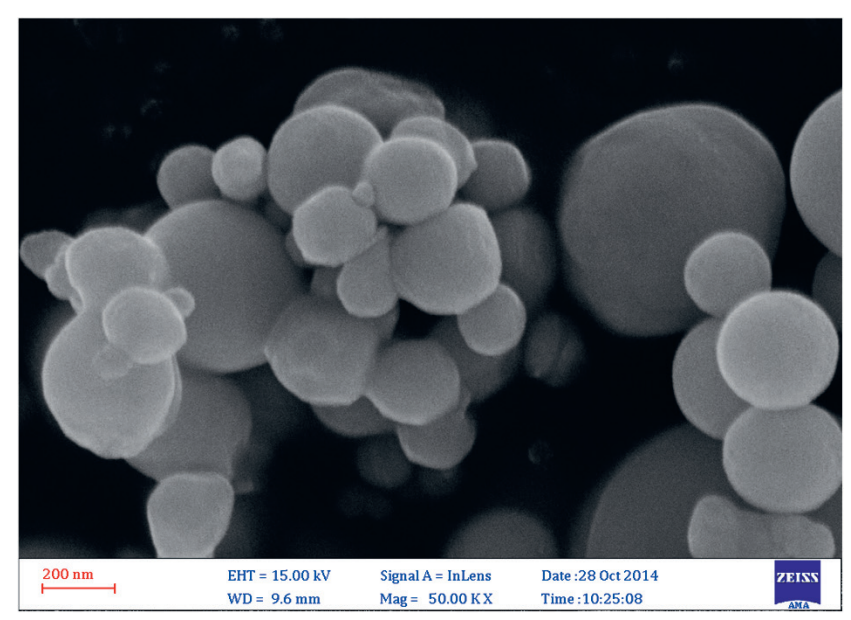

Fig. 3. Scanning electron microscopy (SEM) image of silver nanoparticles (AgNPs); bars indicate $200 \mathrm{~nm}$

\section{Body weight}

There were no significant differences $(p>0.05)$ in mean body weight between the treated groups and the control group (data not shown).

\section{Sperm parameters}

Table 1 shows the effect of AgNPs on sperm parameters. A significant reduction in sperm vitality $(\mathrm{p}<0.05)$, normal sperm morphology $(\mathrm{p}<0.001)$ and sperm count ( $\mathrm{p}<0.0001)$ was observed in the AgNP-3 group, while there were no significant differences in sperm motility in the AgNP-injected groups compared to the control rats.

\section{Testicular histomorphometry}

The data showed a significant reduction in the number of spermatogonia, Sertoli and Leydig cells in the AgNP-2 and AgNP-3 groups ( $\mathrm{p}<0.05)$, whereas there were no significant differences in the number of spermatocytes and spermatid cells among the 4 study groups (Fig. 4).

\section{Parameters of seminiferous tubules}

We observed a significant reduction in the diameter, circumference and mean area of seminiferous tubules in the AgNP-2 group compared to the control group ( $\mathrm{p}<0.001)$ (Table 2).

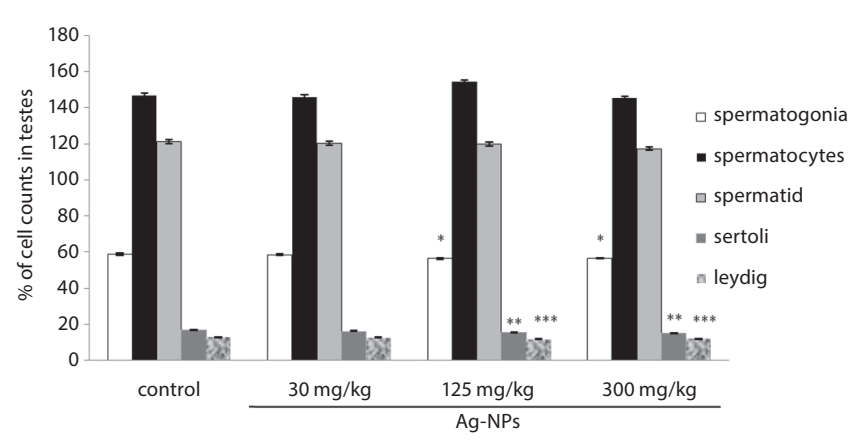

Fig. 4. The effect of silver nanoparticles (AgNPs) on testicular histomorphometry (mean $\pm \mathrm{SD}$ )

${ }^{*} p<0.01,{ }^{* *} p<0.001,{ }^{* * *} p<0.0001$ : (compared to controls); results presented as mean \pm standard deviation (SD).

\section{Assessment of sperm DNA and chromatin}

Sperm DNA and chromatin assay is shown in Fig. 5. As can be seen in Fig. 6, there were no significant differences between the experimental and control groups regarding $\mathrm{AB}, \mathrm{TB}$ and $\mathrm{AO}$ staining $(\mathrm{p}>0.05)$.

\section{Discussion}

The main purpose of the present study was to evaluate the effect of different concentrations of AgNPs on sperm parameters and chromatin, and testicular histomorphometry. Our in vivo data revealed that AgNPs could reduce sperm parameters, such as normal sperm morphology, sperm vitality and sperm count. Silver nanoparticles caused a decrease in Sertoli and Leydig cell number. To better understand the role of AgNPs, we observed the 
Table 1. The effect of AgNPs on sperm parameters

\begin{tabular}{|l|l|l|c|}
\multicolumn{1}{|c|}{ Parameters } & Control & $30 \mathrm{mg} / \mathrm{kg}$ & $125 \mathrm{mg} / \mathrm{kg}$ \\
\hline Motility [\%] & $62.8 \pm 3.03$ & $44.4 \pm 8.41$ & $53.8 \pm 4.18$ \\
\hline Vitality [\%] & $96.7 \pm 0.56$ & $94.5 \pm 0.71$ & $97 \pm 0.72$ \\
\hline Normal morphology [\%] & $96.7 \pm 0.56$ & $92.5 \pm 0.75$ & $93.5 \pm \pm 0.5$ \\
\hline Sperm count $[\times 10 \% \mathrm{~mL}]$ & $53.1 \pm 1.30$ & $51.6 \pm 1.48$ & $89.5 \pm 1.87^{* *}$ \\
\hline
\end{tabular}

AgNPs - silver nanoparticles; ${ }^{*} \mathrm{p}<0.05 ;{ }^{* *} \mathrm{p}<0.001 ;{ }^{* * *} \mathrm{p}<0.0001$ (compared to controls); results presented as mean \pm standard deviation (SD).

Table 2. The effect of AgNPs on seminiferous tubule parameters in testes

\begin{tabular}{|l|c|c|c|}
\multicolumn{1}{|c|}{ Feature } & Control & $30 \mathrm{mg} / \mathrm{kg}$ & $125 \mathrm{mg} / \mathrm{kg}$ \\
\hline Area $\left[\times 10^{-8} \mathrm{~m}^{2}\right]$ & $11.6 \pm 0.19$ & $11.27 \pm 0.17$ & $10.44 \pm 0.18^{*}$ \\
\hline Circumference $\left[\times 10^{-3} \mathrm{~m}\right]$ & $1.20 \pm 0.01$ & $1.18 \pm 0.00$ & $1.18 \pm 0.04^{*}$ \\
\hline Mean diameter $\left[\times 10^{-3} \mathrm{~m}\right]$ & $0.36 \pm 0.00$ & $0.36 \pm 0.00$ & $0.34 \pm 0.00^{*}$ \\
\hline
\end{tabular}

AgNPs - silver nanoparticles; ${ }^{*} \mathrm{p}<0.001$ (compared to controls); results presented as mean \pm standard deviation (SD).
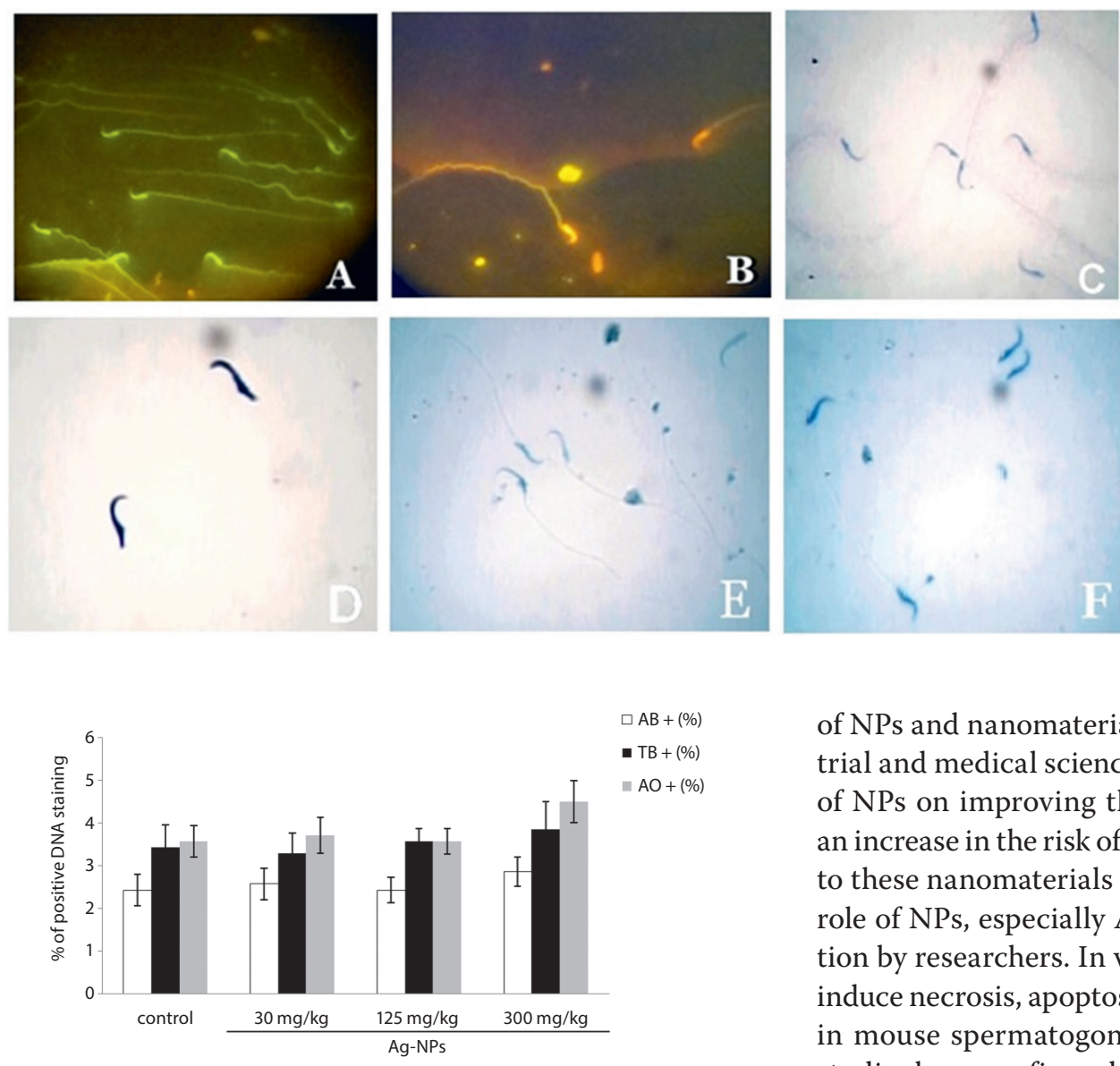

Fig. 6. The effect of AgNPs on sperm nuclear chromatin

AgNPs - silver nanoparticles; AB - anilin blue; TB - toluidine blue; AO - acridine orange; results presented as mean \pm standard deviation (SD).

sperm chromatin packaging by $\mathrm{AB}, \mathrm{TB}$ and $\mathrm{AO}$ staining. Silver nanoparticles did not destroy sperm DNA or chromatin integrity in any of the 3 concentration levels (30, 125 and $300 \mathrm{mg} / \mathrm{kg}$ ).

In the past decade, with the development of nanotechnology, there has been a remarkable increase in application
Fig. 5. Sperm chromatin integrity assay

$A$ - acridine orange $(A O)$, green-stained spermatozoa were considered to be $\mathrm{AO}$ negative (non-denatured DNA); $\mathrm{B}$ - $\mathrm{AO}$ orange-red-stained spermatozoa were considered abnormal (denatured DNA); $\mathrm{C}$ - toluidine blue (TB), unstained or pale blue-stained spermatozoa were considered normal; D - TB, dark bluestained spermatozoa were considered abnormal; E - anilin blue (AB), unstained or pale blue-stained spermatozoa were considered normal; F - AB, dark bluestained spermatozoa were considered abnormal. of NPs and nanomaterials in everyday products in industrial and medical sciences. ${ }^{18}$ Despite the beneficial effects of NPs on improving the quality of life, there is in turn an increase in the risk of human as well as animal exposure to these nanomaterials through various routes. The toxic role of NPs, especially AgNPs, has been under investigation by researchers. In vitro studies have shown that NPs induce necrosis, apoptosis and mitochondrial dysfunction in mouse spermatogonia stem cells. ${ }^{19}$ Similarly, in vivo studies have confirmed the in vitro observations and have suggested that the deleterious effects of NPs are related to their chemical composition, size and dosage. ${ }^{20}$

The i.p. route is one of the most neglected routes for testing AgNPs toxicity. The i.p. route delivers substances into circulation faster than the oral route. In this study, rats were treated with AgNPs at concentrations of 30, 125 and $300 \mathrm{mg} / \mathrm{kg}$. These dosage levels were selected based on a previous 28 -day oral toxicity study by Kim et al. ${ }^{21}$ The pharmacokinetics of substances administered i.p. is more similar to this seen after oral administration. ${ }^{22}$ 
Consistent with previous reports, our results showed a significant reduction in sperm function in a dose-dependent manner. A high dose of AgNPs could affect conventional sperm characteristics, such as normal morphology and viability. Sleiman et al. indicated that AgNPs decreased sperm parameters in the pre-pubertal period of rats. ${ }^{23}$ Sperm count is one of the most sensitive tests for spermatogenesis, since it gives the cumulative result of all stages of sperm production and is highly correlated with fertility.

Silver nanoparticles could damage sperm membranes and/or penetrate the cells, subsequently increasing free radicals, including ROS, that cause membrane lipid peroxidation and, in consequence, loss of motility and viability, and can injure the sperm membrane and flagellum structure, eventually leading to sperm motility and morphology perturbation. . $^{6,194}$

Importantly, based on our data, AgNPs caused a significant reduction in testicular morphological characteristics, such as the diameter, area and circumference of seminiferous tubules, at a concentration of $125 \mathrm{mg} / \mathrm{kg}$, compared to the control group. In contrast to our result, Gromadzka-Ostrowska et al. showed a significant increase in the testicular morphology of animals treated with $200 \mathrm{~nm}$ of AgNPs at a dose of $5 \mathrm{mg} / \mathrm{kg} 28$ days after intravenous injection. ${ }^{10}$ Furthermore, Miresmaeili et al. showed that there were no significant changes in seminiferous tubule diameter in animals treated with oral AgNP administration at doses of 25,50,100, and $200 \mathrm{mg} / \mathrm{kg}$ after 48 days (the time period of spermatogenesis in rats). ${ }^{25}$ Many in vivo studies have shown that chemicals, hypoxia or metals such as chromium, cadmium or lead can decrease the diameter of seminiferous tubule epithelial cells. ${ }^{10}$ Accordingly, it seems that AgNPs have a dual effect on testicular morphological characteristics: at higher concentrations they reduce these features, and at lower doses increase them. Intraperitoneal injection of AgNPs might influence the endocrine system, and, consequently, may manipulate endogenous testosterone and estradiol levels; hence, these endocrine alterations might protect seminiferous tubules at high concentrations. ${ }^{23}$

Moreover, a histological evaluation of the testis tissue indicated a significant decrease in the mean number of spermatogonia, Leydig and Sertoli cells at doses of 125 and $300 \mathrm{mg} / \mathrm{kg}$ compared to controls. In support of our finding, Miresmaeili et al. showed a significant decrease in the number of primary spermatocyte and spermatid cells at doses of 50, 100 and $200 \mathrm{mg} / \mathrm{kg}$. ${ }^{25}$ It has been shown that AgNPs markedly decreased spermatogonial stem cell proliferation by some intracellular pathways. ${ }^{26}$

Silver nanoparticles, like other NPs, can damage the DNA structure of Leydig cells, leading to apoptosis. During spermatogenesis, histones are replaced by protamines in the nuclear DNA. Anilin blue can determine the protamines deficiency in sperm chromatin. The presence of disulfide bonds in the chromatin of mature sperm cells can prevent the denaturation of DNA, and TB can measure the rate of sperm nuclear chromatin condensation and DNA fragmentation via binding to DNA phosphate. Purple sperm heads showed DNA damage. Acridine orange is a fluorescence probe for the detection of denatured or single-stranded DNA in spermatozoa, and red fluorescence in sperm heads showed DNA damage or altered chromatin structure in sperm cells. ${ }^{16}$ Our study also showed no significant changes in chromatin integrity.

Our results were in agreement with the findings of various studies which reported no significant correlation between sperm chromatin condensation and sperm parameters, including motility, vitality, normal morphology, and sperm count. ${ }^{27,28}$ In contrast, other studies reported significant correlations between sperm DNA fragmentation and poor sperm morphology. ${ }^{29,30}$

\section{Conclusions}

Collectively, these findings indicate the effect of AgNPs on the male reproductive system. Normal sperm morphology and viability was disrupted by AgNPs. Silver nanoparticles at a high concentration $(300 \mathrm{mg} / \mathrm{kg})$ also reduced the number of testicular tubules, and spermatogonia, Sertoli and Leydig cells. Spermatozoa DNA was not affected by AgNPs, which needs more investigation on an intracellular level. The in vitro results indicated that the harmful effect of AgNPs is related to their chemical composition, size, dosage, means of administration, and duration of exposure. ${ }^{31}$ Further investigation is necessary to clarify the mechanisms of NP-induced male reproductive dysfunction.

Given these findings, we can conclude that AgNPs pose a potential risk to male fertility, depending on their dose, and taking into consideration the protective role of AgNPs in relation to some parameters, a study over a long period of time should be carried out in our future work.

\section{References}

1. Chaudhry Q, Scotter M, Blackburn J, et al. Applications and implications of nanotechnologies for the food sector. Food Addit Contam Part A Chem Anal Control Expo Risk Assess. 2008;25(3):241-258.

2. Chaloupka K, Malam Y, Seifalian AM. Nanosilver as a new generation of nanoproduct in biomedical applications. Trends Biotechnol. 2010; 28(11):580-588.

3. Sibbald RG, Contreras-Ruiz J, Coutts $P$, Fierheller M, Rothman A, Woo K. Bacteriology, inflammation and healing: A study of nanocrystalline silver dressings in chronic venous leg ulcers. Adv Skin Wound Care. 2007;20(10):549-558.

4. Ahamed M, Alsalhi MS, Siddiqui M. Silver nanoparticle applications and human health. Clin Chim Acta. 2010;411(23-24):1841-1848.

5. Choi JE, Kim S, Ahn JH, et al. Induction of oxidative stress and apoptosis by silver nanoparticles in the liver of adult zebrafish. Aquat Toxicol. 2010;100(2):151-159.

6. Yoshida Y, Itoh N, Saito Y, Hayakawa M, Niki E. Application of water-soluble radical initiator, 2, 2'-azobis-[2-(2-imidazolin-2-yl) propane] dihydrochloride, to a study of oxidative stress. Free Radic Res. 2004;38(4): 375-384.

7. Schrand AM, Rahman MF, Hussain SM, Schlager JJ, Smith DA, Syed AF. Metal-based nanoparticles and their toxicity assessment. Wiley Interdiscip Rev Nanomed Nanobiotechnol. 2010;2(5):544-568. 
8. Pothuraju R, Kaul G. Effect of silver nanoparticles on functionalities of buffalo (Bubalus bubalis) spermatozoa. Adv Sci Eng Med. 2013;5(2):91-95

9. Talebi AR, Khorsandi L, Moridian M. The effect of zinc oxide nanoparticles on mouse spermatogenesis. J Assist Reprod Genet. 2013;30(9): 1203-1209.

10. Gromadzka-Ostrowska J, Dziendzikowska K, Lankoff A, et al. Silver nanoparticles effects on epididymal sperm in rats. Toxicol Lett. 2012; 214(3):251-258.

11. Pourhamzeh M, Mahmoudian ZG, Saidijam M, Asari MJ, Alizadeh Z. The effect of silver nanoparticles on the biochemical parameters of liver function in serum, and the expression of caspase- 3 in the liver tissues of male rats. Avicenna J Med Biochem. 2016;4(2):e35557.

12. Sobczak-Kupiec A, Malina D, Zimowska M, Wzorek Z. Characterization of gold nanoparticles for various medical application. Dig J Nanomater Biostruct. 2011;6(2):803-808.

13. Shokri S, Hemadi M, Bayat G, Bahmanzadeh M, Jafari-Anarkooli I, Mashkani B. Combination of running exercise and high dose of anabolic androgenic steroid, nandrolone decanoate, increases protamine deficiency and DNA damage in rat spermatozoa. Andrologia. 2014;46(2):184-190.

14. Bahmanzadeh M, Vahidinia A, Mehdinejadiani S, Shokri S, Alizadeh Z. Dietary supplementation with astaxanthin may ameliorate sperm parameters and DNA integrity in streptozotocin-induced diabetic rats. Clin Exp Reprod Med. 2016;43(2):90-96.

15. Bahmanzadeh M, Abolhassani F, Amidi F, Sh E, Salehi M, Abbasi M. The effects of nitric oxide synthase inhibitor (L-NAME) on epididymal sperm count, motility and morphology in varicocelized rat. Daru. 2008;16(1):23-28.

16. Talebi AR, Khalili MA, Hossaini A. Assessment of nuclear DNA integrity of epididymal spermatozoa following experimental chronic spinal cord injury in the rat. Int J Androl. 2007;30(3):163-169.

17. Mahmoudian ZG, Sohrabi M, Lahoutian H, Javad M. Histological alterations and apoptosis in rat liver following silver nanoparticle intraorally administration. Entomol App/ Sci Lett. 2016;3(5):27-35.

18. Beer C, Foldbjerg R, Hayashi Y, Sutherland DS, Autrup H. Toxicity of silver nanoparticles - nanoparticle or silver ion? Toxicol Lett. 2012;208(3): 286-292. doi: 10.1016/j.toxlet.2011.11.002

19. Braydich-Stolle L, Hussain S, Schlager JJ, Hofmann M-C. In vitro cytotoxicity of nanoparticles in mammalian germline stem cells. Toxicol Sci. 2005;88(2):412-419.
20. Takeda K, Suzuki K, Ishihara A, et al. Nanoparticles transferred from pregnant mice to their offspring can damage the genital and cranial nerve systems. J Health Sci. 2009;55(1):95-102.

21. Kim YS, Song MY, Park JD, et al. Subchronic oral toxicity of silver nanoparticles. Part Fibre Toxicol. 2010;7:20.

22. Elkhawass EA, Mohallal ME, Soliman MF. Acute toxicity of different sizes of silver nanoparticles intraperitonally injected in BALB/c mice using two toxicological methods. Int J Pharm Pharm Sci. 2014;7(1):94-99.

23. Sleiman HK, Romano RM, Oliveira CA, Romano MA. Effects of prepubertal exposure to silver nanoparticles on reproductive parameters in adult male Wistar rats. J Toxicol Environ Health A. 2013;76(17): 1023-1032. doi: 10.1080/15287394.2013.831723

24. Aziz N, Saleh RA, Sharma RK, et al. Novel association between sperm reactive oxygen species production, sperm morphological defects and the sperm deformity index. Fertil Steril. 2004;81(2):349-354.

25. Miresmaeili SM, Halvaei I, Fesahat F, Fallah A, Nikonahad N, Taherinejad $M$. Evaluating the role of silver nanoparticles on acrosomal reaction and spermatogenic cells in rat. Iran J Reprod Med. 2013;11(5):423.

26. Braydich-Stolle LK, Lucas B, Schrand A, et al. Silver nanoparticles disrupt GDNF/Fyn kinase signaling in spermatogonial stem cells. Toxicol Sci. 2010;116(2):577-589.

27. Hammadeh M, Zeginiadov T, Rosenbaum P, Georg T, Schmidt W, Strehler E. Predictive value of sperm chromatin condensation (aniline blue staining) in the assessment of male fertility. Arch Androl. 2001;46(2):99-104

28. Salsabili N, Mehrsai A, Jalalizadeh B, Pourmand G, Jalaie S. Correlation of sperm nuclear chromatin condensation staining method with semen parameters and sperm functional tests in patients with spinal cord injury, varicocele and idiopathic infertility. Urol J. 2009;3(1): 32-37.

29. Franken D, Franken C, De La Guerre H, De Villiers A. Normal sperm morphology and chromatin packaging: Comparison between aniline blue and chromomycin A3 staining. Andrologia. 1999;31(6): 361-366.

30. Mehdi M, Khantouche L, Ajina M, Saad A. Detection of DNA fragmentation in human spermatozoa: Correlation with semen parameters. Andrologia. 2009;41(6):383-386.

31. Castellini C, Ruggeri S, Mattioli S, et al. Long-term effects of silver nanoparticles on reproductive activity of rabbit buck. Syst Biol Reprod Med. 2014;60(3):143-150. 